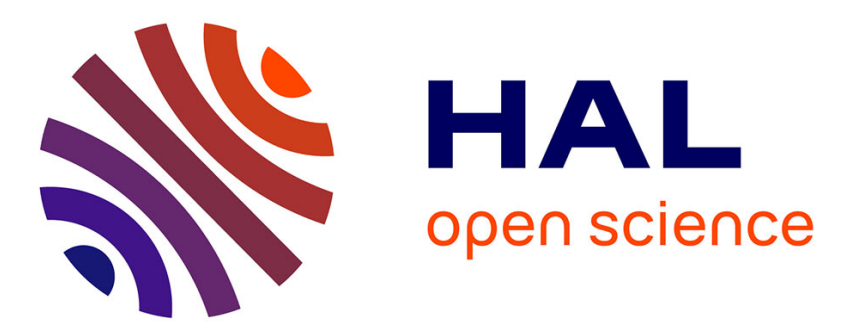

\title{
Buffer dynamic management for energy-aware network
}

Wael Zouaoui, Yann Labit, Carolina Albea-Sanchez

\section{To cite this version:}

Wael Zouaoui, Yann Labit, Carolina Albea-Sanchez. Buffer dynamic management for energy-aware network. 10th International Conference on Network and Service Management, Nov 2014, Rio de Janeiro, Brazil. hal-01068667v2

\section{HAL Id: hal-01068667 \\ https://hal.science/hal-01068667v2}

Submitted on 26 Nov 2014

HAL is a multi-disciplinary open access archive for the deposit and dissemination of scientific research documents, whether they are published or not. The documents may come from teaching and research institutions in France or abroad, or from public or private research centers.
L'archive ouverte pluridisciplinaire HAL, est destinée au dépôt et à la diffusion de documents scientifiques de niveau recherche, publiés ou non, émanant des établissements d'enseignement et de recherche français ou étrangers, des laboratoires publics ou privés. 


\section{Buffer dynamic management for energy-aware network}

\author{
Wael Zouaoui \\ CNRS, LAAS, \\ 7, avenue du Colonel Roche \\ F- 31400 Toulouse France \\ Univ de Toulouse, UPS, LAAS, \\ F- 31400 Toulouse France \\ wzouaoui@laas.fr
}

\author{
Yann Labit \\ CNRS, LAAS, \\ 7, avenue du Colonel Roche \\ F- 31400 Toulouse France \\ Univ de Toulouse, UPS, LAAS, \\ F- 31400 Toulouse France \\ ylabit@laas.fr
}

\author{
Carolina Albea \\ CNRS, LAAS, \\ 7, avenue du Colonel Roche \\ F- 31400 Toulouse France \\ Univ de Toulouse, UPS, LAAS, \\ F- 31400 Toulouse France \\ calbea@laas.fr
}

\begin{abstract}
Energy is a major issue in networks especially in local network implementation. Some technological solutions have been developed to treat this problem e.g. Re-engineering, Dynamic Adaptation, Smart Sleeping. We present an original and alternative adaptive solution from a proposed extended Adaptive Link Rate (ALR) module, which relates energy and router queuelength. Applying a controller already proposed by authors, the router queue-length is adapted in order to follow a reference given by the extended ALR module, that guarantees an energy reduction. The approach is validated in the network simulator "NS-2". Within ADREAM building at LAAS-CNRS, this "control green" will allow to improve energy consumption regarding traffic load and available energy in communication networks.
\end{abstract}

Keywords-Green Network, Adaptive system, Quality of Service, Energy, ADREAM, Adaptive Link Rate.

\section{INTRODUCTION}

Since few years, the number of communication system users has significantly increased, thereby energy consumption have also increased. According to [1], the number of Internet users reached 2.4 billion people in 2012. Consequently, the energy consumption in communication networks represents $5.5 \%$ of annual world electricity production and a rate of $2 \%$ of carbon dioxide emissions $\left(\mathrm{CO}_{2}\right)$. The perspective for 2015 , with probably 2.8 billion of Internet users, is to achieve a traffic 4 times larger and to double energy consumption (approximately 25\% larger per year) [2]. This issue requires to research new ways to reduce energy consumption.

In wired networks, energy reduction often diminishes Quality of Service (QoS). This performance, energy reduction trade-off requires to define an efficient strategy to decrease the network energy consumption, what is a real challenge.

Some interesting hardware and protocol solutions have been proposed to reduce energy consumption in communication networks. In [3], the author's survey mention some techniques which aim is to improve energy efficiency of computing and network resources. They classify these methods as follows:

a) Re-Engineering: This approach aims at modifying and re-engineering network architectures to use more energyefficient elements (green routers, switches ...). This solution is complex and expensive due to fundamental changes in network [4], [5], [6], [7]. b) Dynamic Adaptation: This solution modulates network resources capacities based on varying traffic loads and service requirements. "Performance Scaling" and "Idle Logic" use this approach with the goal of reducing energy consumption in network [8], [9], [10], [11].

c) Smart Sleeping: Energy consumption is reduced by switching off some network equipment when they are inactive, and to smartly wake them up only if necessary. There are two kinds of smart sleeping: "on/off and sleeping technology", to switch off the inactive or idle network components [12], [13]; and "proxying technology" that uses a proxy to answer to nonurgent messages instead of employing a node, which is wakedup when required [14], [15].

In the industry domain, some important companies in the sector have already started a "green revolution" (development of "green" network equipments). Among these companies, we cite the following ones: Cisco (EnergyWise) that focuses on reducing power utilization of all devices connected to a network [16]; Alcatel-Lucent (GreenTouch) based on all-optical networking systems with the goal to master the technology for future green networks [17]; IBM (Big-Green), which goal is to reduce data centers' energy consumption [18]; ICT4EE (Information and Communication Technologies for Energy Efficiency) forum (GeSI, DigitalEurope, JBCE and Europe TechAmerica), whose objective is the enhancement of energy efficiency in ICT systems [19]; NetGear that offers a wide range of innovative routers and energy-aware switches [20] and Hewlett-Packard, Intel, Microsoft, Phoenix Technologies, Toshiba (Advanced Configuration and Power Interface) that provides an open standard for device configuration and power management by the Operating System [21].

In this paper, an extended ALR module is proposed to show the existing relationship between energy and router queuelength. Then, we apply a control algorithm developed by us [22] to a router. This control adapts the traffic that crosses a node by using length-queue where packets are temporarily stored. The adapted length-queue converges to the suboptimal reference given by ALR module, which guarantees an energy reduction. The choice of the selected nodes is a problem in itself, and it is not the focus of this work. The proposed solution is validated in NS-2 [23] and exploited in ADREAM (Architectures for Dynamic Resilient Embedded 
Autonomous and Mobile systems) project (building devoted to cyber-physical systems at LAAS-CNRS, figure 1). We consider two functioning modes (figure 2):

- Normal mode: During a daytime, the system administrator ensures the proper functioning of the communication system, the introduction of new materials, computer security and daily network administration. With no software or hardware anomaly, the administrator faces a normal operating mode.

- The energy aware mode: to make an intelligent management computer system (from an energy point of view), the administrator programs the energy aware mode for a sensible time interval (night) and/or in cooperation with users (e.g. time slot $12 \mathrm{H}-14 \mathrm{H})$. This mode of operation is a degraded mode (e.g. trade off between energy saving and quality of service parameters such as transmission delay information).

Likewise, energy consumption measures are given by ECOFEN [24] (implemented module in NS-2).

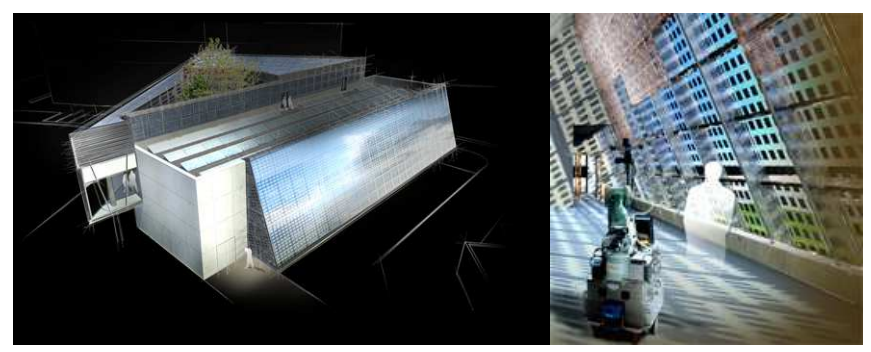

Fig. 1. ADREAM building at LAAS-CNRS.

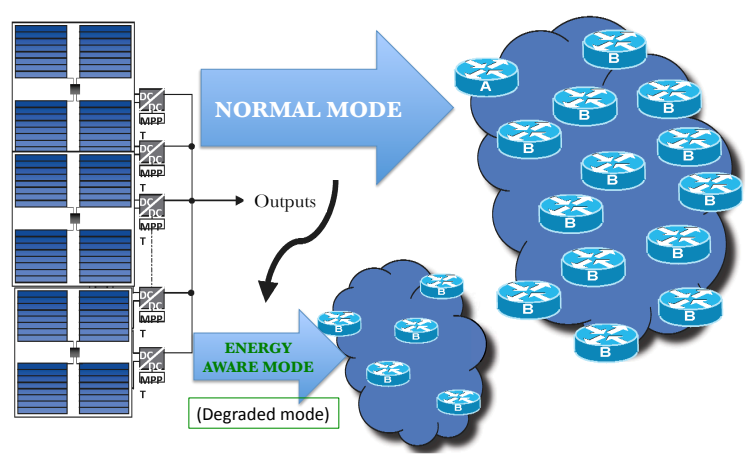

Fig. 2. The two modes (normal and degraded).

\section{ALR MODEL AND ECOFEN MODULE}

Firstly, lets model the energy consumption of the network equipment. For this purpose, we consider a router model designed in NS-2. NS-2 is currently one of the most widely used simulators in the network community [23]. This simulator allows us to simulate large networks with realistic traffic and to obtain QoS measures.

The ECOFEN module (Energy Consumption Model for End-to-end Networks) was developed in NS-2. This module provides the instantaneous energy consumption of each equipment taking into account the traffic and the type of employed equipment (router, switch, network card, etc.) [24]. This module is based on the ALR model (Adaptive Link Rate) which adapts transmission rates depending on link use-rate.
Lets take previous model for studying energy consumption in a router. For this issue, we adapt input traffic, $w_{n}$, of the controlled node. We artificially and temporarily delay a certain number of packets in a buffer to a pre-defined value $q_{\text {ref }}$. The length-queue reference $q_{r e f}$ is given according to the input traffic in the router. This selection may provide some problems in network QoS, in terms of "delay". In other words, there is an energy consumption reduction and transmission delay tradeoff. From the ECOFEN model given in [24], the new extended model is

$$
P= \begin{cases}P_{0} & \text { if } w_{n}=0 \\ a_{1} w_{n}+P_{1}-b q_{r e f} & \text { if } \left.\left.w_{n} \in\right] 0 ; W_{1}\right] \\ \vdots & \\ a_{i} w_{n}+\left(P_{i}-a_{i} W_{i-1}\right)-b q_{r e f} & \text { if } \left.\left.w_{n} \in\right] W_{i-1} ; W_{i}\right] \\ \vdots & \\ a_{N} w_{n}+\left(P_{n}-a_{N} W_{N-1}\right)-b q_{r e f} & \text { if } \left.\left.w_{n} \in\right] W_{N-1} ; W_{N}\right]\end{cases}
$$

with :

- $\quad a_{i} \in \mathbb{R}^{+}$: the slope of the different linear section.

- $w_{n} \in \mathbb{R}^{+}$: the input traffic of each network element.

- $W_{i} \in \mathbb{R}^{+}:$the use-rate thresholds.

- $\quad P_{i} \in \mathbb{R}^{+}$: the start power of each different stage.

- $\quad q_{\text {ref }} \in \mathbb{R}^{+}$: the queue length reference.

- $\quad b \in \mathbb{R}^{+}$: a coefficient of the queue length reference $q_{\text {ref }}$.

Figure 3 shows the modified power model for a router. In this model, the input traffic depends on the consumed energy by the network element. Therefore, a reliable adaptation of the queue length reference is mandatory in order to reduce energy consumption with QoS guarantees. This selection of

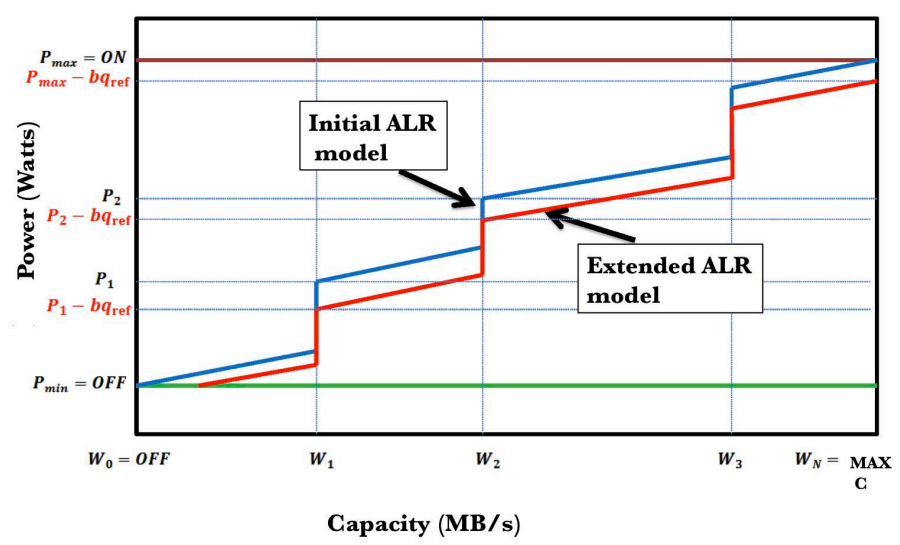

Fig. 3. Modified ALR model of power consumption in a network element (router model).

$q_{\text {ref }}$ depends on two key parameters:

- the update time-window for the queue length reference.

$$
T_{q_{\text {ref }}}=\frac{T_{w}}{\beta},
$$

where $T_{w}$ is the time-window related to the node input traffic. $\beta$ is the number of changes of the value of $q_{\text {ref }}$ during the period $T_{w}$. This allows to update more or less frequently $q_{r e f}$ 
depending on the traffic variability.

- The queue length reference

$$
q_{\text {ref }}=\frac{\hat{w}_{n}}{\gamma},
$$

where $\hat{w}_{n}$ is the average input traffic on a fixed time-window $T_{w} . \gamma$ is a step (weighting coefficient) for adapting the amplitude of the reference function $w_{n}$.

Remark. The judicious choice of the average input traffic $\hat{w}_{n}$, on a fixed time window $T_{w}$, take into account the high variability of this traffic.

\section{Analytic \& Simulation Results}

The proposed control law in discrete-time is:

$$
u_{k}=\operatorname{sat}_{0}^{C}\left\{u_{k-1}+K x_{k}\right\},
$$

where $K=\left[\begin{array}{ll}K_{1} & K_{2}\end{array}\right]$ are control gains computed following the mechanism proposed in [22]. $x_{k}=\left[\begin{array}{ll}e_{k} & e_{k-1}\end{array}\right]^{T}$, where $e_{k}$ is the error between queue-length reference and queue-length in time-period $k$. The high-bound saturation $C$ depends on network capacity. We choose: $C=25000 \mathrm{pkts} / \mathrm{s}, T_{s}=0.05 \mathrm{~s}$. From [22], it comes that: $K_{1}=-21$ and $K_{2}=20$. To validate this approach, we take a simple topology composed of two nodes and one source, as shown in Figure 4. In Figure 4 , we show the traffic rate crossing a switch located in the ADREAM building of LAAS-CNRS in the daytime. Several simulations with different values of $\beta$ and $\gamma$ are done to select the best couple that gives the maximum of power gain $\left(G_{W}\right)$ and maximum ratio of time that power gain is minimized $\left(T_{G_{W}}\right)$. Taking 21 values for $\gamma$ from 500 to 1500 with a pitch of 50. For each value of $\gamma$, we assign 8 values for the update window $\beta \in[1,8]$. In Figure 5, we present the power gain and the ratio of time when we have power gain for different values of $\beta$ function of $\gamma$. It is clearly seen the influence of theses parameters on energy consumption. The best value of $G_{W}$ and $T_{G_{W}}$ are achieved with the couple $(\beta, \gamma)=(8,850)$.

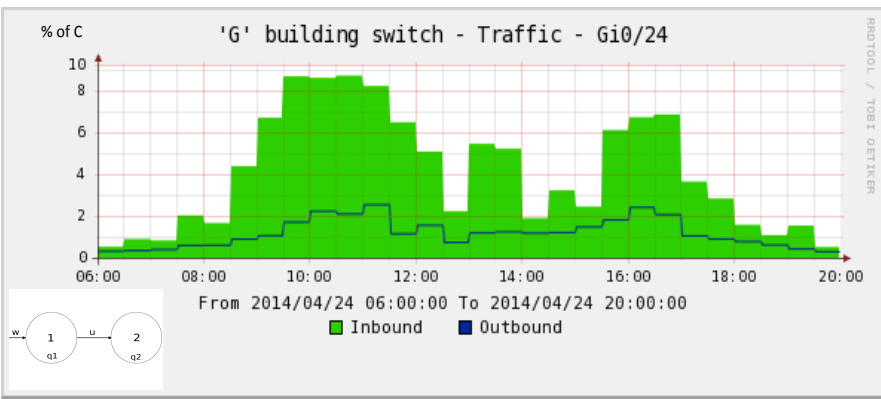

Fig. 4. ADREAM switch traffic

After choosing the couple $(\beta, \gamma)$, some analog simulations of the node 4 are performed here. In Figure 6, the evolution of the input traffic similar to the traffic rate in Figure 4 and the output traffic calculated from the control law are presented. Likewise, it is shown in Figure 7, the queue length evolution. Note that the network traffic is unsaturated, so losses are avoided. Figure 8 (up) shows the power consumption with and without control law. Note, there is a significant improvement in power consumption from $9: 30 h$ to $11: 30 h$ and from $14: 30 h$ to $18: 30 h$ which represents $60 \%$ of the total
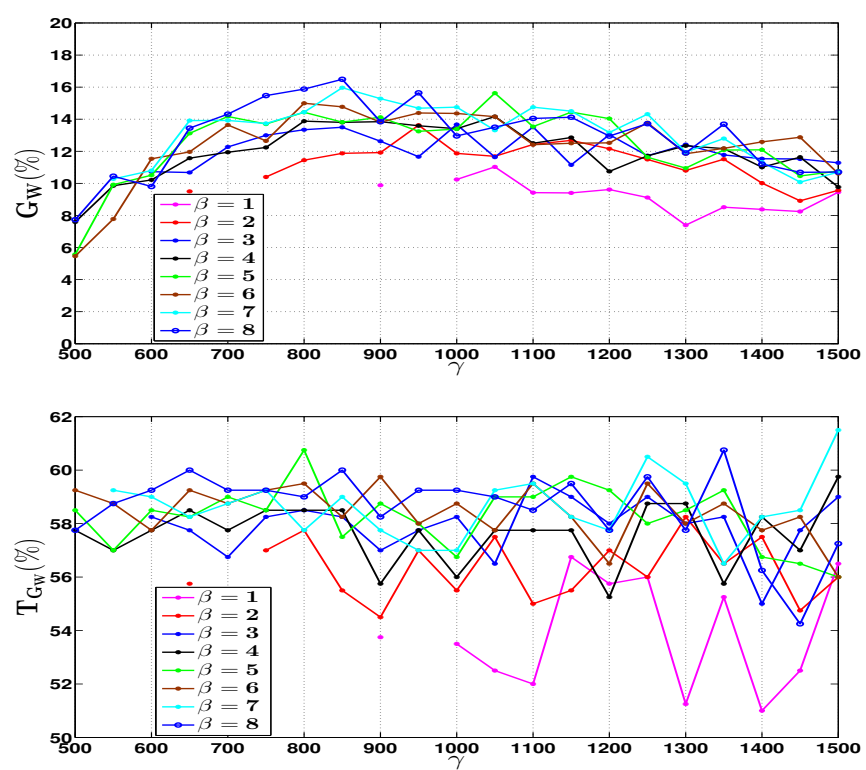

Fig. 5. Power gains (up) and Time power gain (bottom) with differents values of $\beta$ and $\gamma$

simulation time $(20 h)$. During this time period, packets are stored in the queue (with size $q_{r e f}$ ), the output traffic is smaller than the input traffic. Moreover, power is smaller than a power threshold, hence the reduction of the energy consumption. Given the ALR model, if the output traffic is smaller than a threshold $W_{i}$ (see Figure 3), the consumed energy will be smaller than without control. On the other hand, for the others periods of time, queue length releases packets already stored. Hence, the output traffic is larger than the input traffic, what leads to a slight energy overconsumption, the difference is less than the reduction.

Note that the minimum power consumption of the ALR model is $P_{0}=155 \mathrm{~W}$ (Figure 8). This value is obtained when there is not traffic crossing the controlled node $\left(w_{n}=\right.$ $0 \mathrm{Mb} / \mathrm{s}$ ). This is a minimum power consumption of the operational node without traffic.

In Figure 8 (bottom), the two phases of energy consumption (reduced and increased energy consumption) are easily seen. In this simulation, it is shown that the global energy consumption for a time period of $20 h$ is reduced $16.49 \%$ (energy gain) in the ALR model. The ratio between reduced and increased energy consumption is up to $60 \%$.

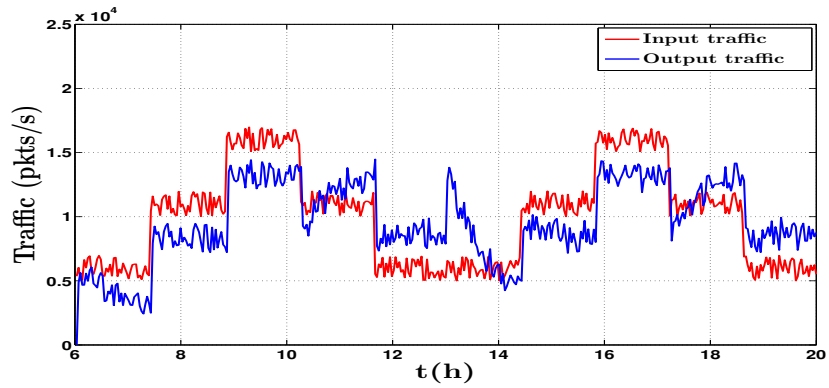

Fig. 6. Input (red) and output (blue) traffic 


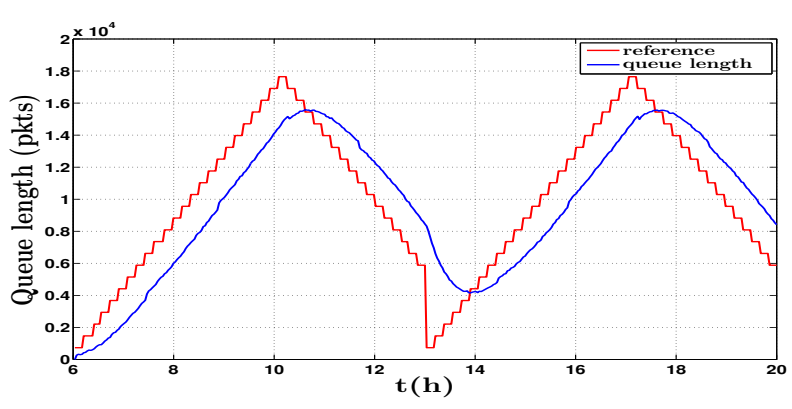

Fig. 7. Queue length evolution (blue) and queue length reference (red).
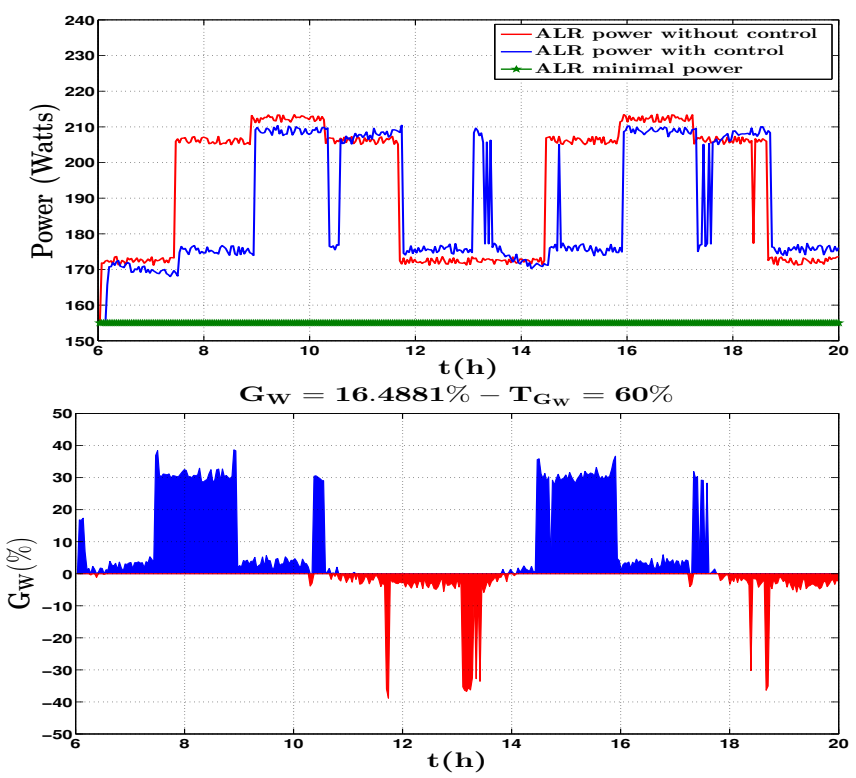

Fig. 8. Power consumption (up) with (blue) and without (red) flow control, on ALR model of a network node - Power gain (Bottom)).

\section{CONCLUSION}

In this article, an alternative adaptive solution for reducing energy consumption in networks has been presented. This solution is developed from a proposed ALR that relates router energy and queue-length. By using a control protocol developed by us, we achieve to adapt the router queue-length in such a way that it converges to the reference given by the extended ALR model, and thus, energy is reduced. Simulations in and NS-2 validate our algorithm. The shown energy gain let to call our algorithm "control green".

\section{REFERENCES}

[1] J. Koomey, "Worldwide electricity used in data centers," Environ. Res. Lett. 3 034008, 2008.

[2] Z. Zhang, A. S. Ackerman, G. Feingold, S. Platnick, R. Pincus, and H. Xue, "Effects of cloud horizontal inhomogeneity and drizzle on remote sensing of cloud droplet effective radius: Case studies based on large-eddy simulations," Journal of Geophysical Research: Atmospheres, vol. 117, no. D19, pp. n/a-n/a, 2012. [Online]. Available: http://dx.doi.org/10.1029/2012JD017655

[3] R. Bolla, R. Bruschi, F. Davoli, and F. Cucchietti, "Energy efficiency in the future internet: A survey of existing approaches and trends in energy-aware fixed network infrastructures," Communications Surveys Tutorials, IEEE, vol. 13, no. 2, pp. 223-244, Second 2011.
[4] M. Baldi and Y. Ofek, "Time for a "greener" internet," in Communications Workshops, 2009. ICC Workshops 2009. IEEE International Conference on, June 2009, pp. 1-6.

[5] J. Noguera and I. Kennedy, "Power reduction in network equipment through adaptive partial reconfiguration," in Field Programmable Logic and Applications, 2007. FPL 2007. International Conference on, Aug 2007, pp. 240-245.

[6] L. Roberts, "A radical new router," Spectrum, IEEE, vol. 46, no. 7, pp. 34-39, July 2009.

[7] M. Gupta and S. Singh, "Using low-power modes for energy conservation in ethernet lans," in INFOCOM 2007. 26th IEEE International Conference on Computer Communications. IEEE, May 2007, pp. 24512455.

[8] M. Yamada, T. Yazaki, N. Matsuyama, and T. Hayashi, "Power efficient approach and performance control for routers," in Communications Workshops, 2009. ICC Workshops 2009. IEEE International Conference on, June 2009, pp. 1-5.

[9] B. Zhang, K. Sabhanatarajan, A. Gordon-Ross, and A. George, "Realtime performance analysis of adaptive link rate," in Local Computer Networks, 2008. LCN 2008. 33rd IEEE Conference on, Oct 2008, pp. 282-288.

[10] S. Nedevschi, L. Popa, G. Iannaccone, S. Ratnasamy, and D. Wetherall, "Reducing network energy consumption via sleeping and rateadaptation," in Proceedings of the 5th USENIX Symposium on Networked Systems Design and Implementation, ser. NSDI'08. Berkeley, CA, USA: USENIX Association, 2008, pp. 323-336. [Online]. Available: http://dl.acm.org/citation.cfm?id=1387589.1387612

[11] C. Gunaratne, K. Christensen, B. Nordman, and S. Suen, "Reducing the energy consumption of ethernet with adaptive link rate (alr)," Computers, IEEE Transactions on, vol. 57, no. 4, pp. 448-461, April 2008.

[12] C. Hu, C. Wu, W. Xiong, B. Wang, J. Wu, and M. Jiang, "On the design of green reconfigurable router toward energy efficient internet," Communications Magazine, IEEE, vol. 49, no. 6, pp. 83-87, June 2011.

[13] K. Christensen, P. Reviriego, B. Nordman, M. Bennett, M. Mostowfi, and J. Maestro, "Ieee 802.3az: the road to energy efficient ethernet," Communications Magazine, IEEE, vol. 48, no. 11, pp. 50-56, November 2010.

[14] Y. Agarwal, S. Savage, and R. Gupta, "Sleepserver: A software-only approach for reducing the energy consumption of pcs within enterprise environments," in Proceedings of the 2010 USENIX Conference on USENIX Annual Technical Conference, ser. USENIXATC' 10. Berkeley, CA, USA: USENIX Association, 2010, pp. 22-22. [Online]. Available: http://dl.acm.org/citation.cfm?id=1855840.1855862

[15] K. Sabhanatarajan, A. Gordon-Ross, M. Oden, M. Navada, and A. George, "Smart-nics: Power proxying for reduced power consumption in network edge devices," in Symposium on VLSI, 2008. ISVLSI '08. IEEE Computer Society Annual, April 2008, pp. 75-80.

[16] Cisco. Energywise. [Online]. Available: http://www.cisco.com/en/US/ $\mathrm{prod} / \mathrm{switches} / \mathrm{ps} 5718 / \mathrm{ps} 10195 /$ white_paper_c11-514539.html

[17] Alcatel-Lucent. The green touch initiative. [Online]. Available: http://www.greentouch.org/index.php?page=home

[18] IBM. The big-green project. [Online]. Available: http://www-03.ibm. com/press/us/en/presskit/21440.wss

[19] ICT4EE. The ict for energy efficiency forum. [Online]. Available: http://www.digitaleurope.org/index.php?id=1145

[20] NetGear. Netgear green. [Online]. Available: http://www.netgear.fr/ about/netgear-green/green-products/default.aspx

[21] ACPI. Advanced configuration and power interface. [Online]. Available: http://www.acpi.info/DOWNLOADS/ACPIspec50.pdf

[22] W. Zouaoui, C. Albea-Sanchez, and Y. Labit, "Dynamic adaptive management of buffer for QoS guarantees and energy reduction," in Computer Software and Applications Conference (COMPSAC), 2014 IEEE 38th Annual, July 2014.

[23] K. Fall and K. Varadhan, "The ns manual," notes and documentation on the software ns2-simulator, 2002, uRL: www.isi.edu/nsnam/ns/.

[24] A. Orgerie, L. Lefèvre, and I. Guérin-Lassous, "Energy-efficient bandwidth reservation for bulk data transfers in dedicated wired networks," The Journal of Supercomputing, pp. 1-28, 2011. 